\title{
COMUNIDADE DE PRÁTICA NO ENSINO SUPERIOR
}

\author{
Vagner Ricardo de Araujo PEREIRA ${ }^{1}$ \\ Carlos Roberto Massao HAYASHI ${ }^{2}$
}

RESUMO: O objetivo deste trabalho é analisar a inserção, no ensino superior, de um recurso da gestão do conhecimento denominado Comunidade de Prática $(\mathrm{CoP})$ ou Comunidade de Aprendizagem Profissional. Para isso, foi realizada uma pesquisa bibliográfica que procurou responder à seguinte questão: quais fatores contribuem para a criação, manutenção e fortalecimento de uma CoP no ensino superior? Os resultados indicam que a inserção de uma $\mathrm{CoP}$ no ensino superior depende de diversos fatores, dentre eles a valorização das relações sociais entre seus membros, o apoio institucional como forma de superar a perda de exclusividade do conhecimento por meio do registro das informações e a necessidade de um facilitador que viabilize e organize as reuniões, bem como estimule a frequência aos encontros. A comunidade de prática no ensino superior pode ocorrer entre professores das áreas específica e pedagógica e entre estudantes, ou ainda, como uma combinação desses atores. Além disso, é possível envolver atores externos, tais como profissionais vinculados a empresas públicas ou privadas. Diversos estudos internacionais indicam resultados bem-sucedidos, entretanto, poucas pesquisas são encontradas no Brasil.

PALAVRAS-CHAVE: Comunidade de Prática (CoP). Gestão do conhecimento. Ensino superior.

\section{INTRODUÇÃO}

O ensino superior é fundamentalmente constituído por conhecimentos práticos, voltados à formação profissional. O estudante deve ser estimulado a avaliar, desafiar e modificar o conhecimento estabelecido, podendo construir novos conhecimentos, por meio de um processo dinâmico (FENWICK; EDWARDS, 2014). Nesse sentido, a participação do estudante deve ser ativa, diferentemente do que ocorre no ensino tradicional, ainda muito presente na realidade das instituições de ensino superior.

Uma renovação educativa é necessária para que diversas estratégias de ensino contribuam para a participação ativa do estudante, como o trabalho em equipes multidisciplinares, o debate sobre problemas interdisciplinares, pesquisa, aproximação do ambiente escolar da realidade profissional, dentre outras. Nesse contexto, a

\footnotetext{
${ }^{1}$ Doutorando em Ciência, Tecnologia e Sociedade. UFSCar - Universidade Federal de São Carlos - Pósgraduação em Ciência, Tecnologia e Sociedade. São Carlos - SP - Brasil. 13565-905. UNIFEB - Centro Universitário da Fundação Educacional de Barretos. Faculdades Unificadas. Barretos - SP - Brasil. 14783-226 - vagnerap2@gmail.com

${ }_{2}^{2}$ UFSCar - Universidade Federal de São Carlos. Centro de Educação e Ciências Humanas Departamento de Ciências da Informação. São Carlos - SP - Brasil. 13565-905 - massao@ufscar.br
} 
comunidade de prática (CoP) pode oferecer algumas contribuições ao trabalho pedagógico.

As comunidades de prática, também conhecidas como comunidades de aprendizagem profissional, são formadas por pessoas engajadas em um processo de aprendizagem coletiva, compartilhando a preocupação de desenvolver algo de forma cada vez melhor, por meio da interação contínua entre seus membros (WENGER, 2015).

Nem toda comunidade pode ser denominada uma comunidade de prática, pois sua constituição depende de três características importantes. $\mathrm{O}$ aspecto do domínio, no qual seus membros têm uma identidade definida devido a um interesse compartilhado, cujas competências os distinguem de outras pessoas, aprendendo um com o outro. $\mathrm{O}$ aspecto da comunidade em si, cujos membros estão engajados em atividades e discussões conjuntas, construindo relações que levam à aprendizagem em determinada organização. E o aspecto relacionado a prática, cujos participantes desenvolvem um conjunto de recursos: “[...] experiências, histórias, ferramentas, maneiras de tratar problemas recorrentes, em suma, uma prática compartilhada." (WENGER, 2015, p.2, tradução nossa).

No ambiente escolar, nota-se que o desenvolvimento profissional não depende exclusivamente do conteúdo para a formação acadêmica, mas também da interação com colegas, leituras e reflexões pessoais. O desenvolvimento profissional não ocorre desvinculado de um referencial teórico e, também, está associado a um contexto (FERREIRA; SILVA, 2014). Nesse sentido, a CoP vem auxiliar a sistematização do processo de aprendizagem, fornecendo uma estrutura para a coleta de dados e o tratamento das informações, tendo em vista a construção de conhecimento.

Em uma comunidade de prática, diversos níveis de participação podem ser encontrados. Eles variam de acordo com o engajamento de cada membro. Os níveis podem variar desde membros muito ativos, com alto nível de discernimento, até membros que não participam diretamente das discussões realizadas pelo núcleo da CoP. Os membros de uma comunidade de prática podem transitar de um nível para outro, considerando seus próprios interesses e engajamento, em determinado momento (FERREIRA; SILVA, 2014).

A implantação, o desenvolvimento e a sobrevivência de uma CoP pode ser abalada pela percepção de seus membros da perda da exclusividade sobre certo conhecimento, o que poderia levar a sua substituição por outra pessoa, pois a detenção 
de um conhecimento exclusivo pode garantir poder e manutenção do emprego em uma organização. Assim, a atividade de uma CoP depende da motivação e do incentivo recebido pelos membros, pois a $\mathrm{CoP}$ pode assumir papeis estratégicos dentro de uma organização, principalmente aquelas que dependem de conhecimento criativo (JANG; KO, 2010).

Como forma de garantir as atividades de uma CoP na organização, avaliações de seu desempenho são necessárias, principalmente em relação ao compromisso de seus membros, pois é a base de recursos para construir o potencial operacional da organização (JANG; KO, 2010).

A estrutura para o novo plano estratégico do Programa de Desenvolvimento das Nações Unidas (UNDP, 2014) destaca que as comunidades de prática devem ultrapassar as fronteiras regionais, cujo sucesso dessas comunidades depende de sua viabilização por meio de orientação do conteúdo e garantia de qualidade, as quais necessitam de recursos adequados. Além disso, deve fortalecer o modelo de comunidade integrada que é desenhada especificamente para evitar a criação de novas temáticas, além daquelas definidas no planejamento estratégico, de forma a não perder o foco, mas fomentar e premiar o engajamento com redes externas, pensando em temáticas cruzadas e trocas interdisciplinares entre as equipes formadas em torno do desenvolvimento de soluções. Diante das expectativas da sociedade, conhecer o funcionamento de CoPs bem como de outras ferramentas da gestão do conhecimento é uma vantagem ao profissional no mercado de trabalho.

Assim, professores envolvidos em tais atividades podem adquirir experiência e orientar os estudantes a buscarem soluções para problemas ou desenvolverem projetos por meio de equipes de trabalho, conhecendo e utilizando a dinâmica da CoP, em busca de compartilhamento de conhecimento e maior engajamento de seus membros, valorizando o papel da tecnologia da informação e da interação entre as pessoas nesse processo.

De acordo com Nonaka e Toyama (2003, p.3, tradução nossa), “[...] a criação de conhecimento é um processo de síntese por meio do qual a organização interage com os indivíduos e o ambiente para transcender contradições emergentes que ela enfrenta." Considerando uma instituição de ensino superior como de aprendizagem e criadora de conhecimento, pode-se encontrar diversas situações em que conflitos podem ocorrer devido à ação e interação entre as pessoas envolvidas, por exemplo, na elaboração de 
projetos, em atividades de sala de aula, na dinâmica do trabalho em equipe, em situações de laboratório, etc.

Nesse processo de interação, as pessoas são fortemente influenciadas pelo contexto, enquanto recriam continuamente seu ambiente por meio das interações sociais e criatividade. Davenport e Prusak (1999, p.117-118) apresentam algumas soluções possíveis para as dificuldades enfrentadas em relação ao compartilhamento de conhecimento, são elas:

- Construir relacionamentos e confiança mútua através de reuniões face a face.

- Estabelecer um consenso através de educação, publicações, trabalho em equipe e rodízio de funções.

- Criar tempo e locais para transferências do conhecimento: feiras, salas de bate-papo, relatos de conferências.

- Avaliar o desempenho e oferecer incentivos ao compartilhamento.

- Educar funcionários para a flexibilidade; propiciar tempo para aprendizado; basear as contratações na abertura a ideias.

- Estimular a aproximação não hierárquica do conhecimento; a qualidade das ideias é mais importante que o cargo da fonte.

- Aceitar e recompensar erros criativos e colaboração; não há perda de status por não se saber tudo.

Líderes escolares e professores deveriam aplicar recursos da gestão do conhecimento como forma de estimular o comprometimento de todos no contexto educacional. A visão de gestão do conhecimento no ambiente escolar não deve ser adquirida somente pelos dirigentes, mas compartilhada entre os professores por meio de contínuas interações entre seus membros, além de promover um clima de confiança, necessária para que ocorra a externalização das informações e do conhecimento, tanto entre indivíduos quanto intergrupais, visando à melhoria das condições de aprendizagem (CHENG, 2013).

O diagrama da Figura 1 representa a dinâmica da gestão do conhecimento em ambiente educacional, proposto por Cheng (2013). Ele aponta quatro aspectos estratégicos para o desenvolvimento organizacional sustentável, a gestão da organização, o ensino e aprendizagem, o suporte ao estudante e as conquistas dos estudantes, cuja relevância dos dados obtidos é atribuída pelo receptor e transformada em informação. Essa informação contextualizada, frente à visão especializada das pessoas envolvidas, fornece uma estrutura de apoio para a análise e incorporação de novas experiências e informações, constituindo a base para a construção de novos conhecimentos (DAVENPORT; PRUSAK, 2000). 
Figura 1 - Diagrama representativo da gestão do conhecimento em escolas

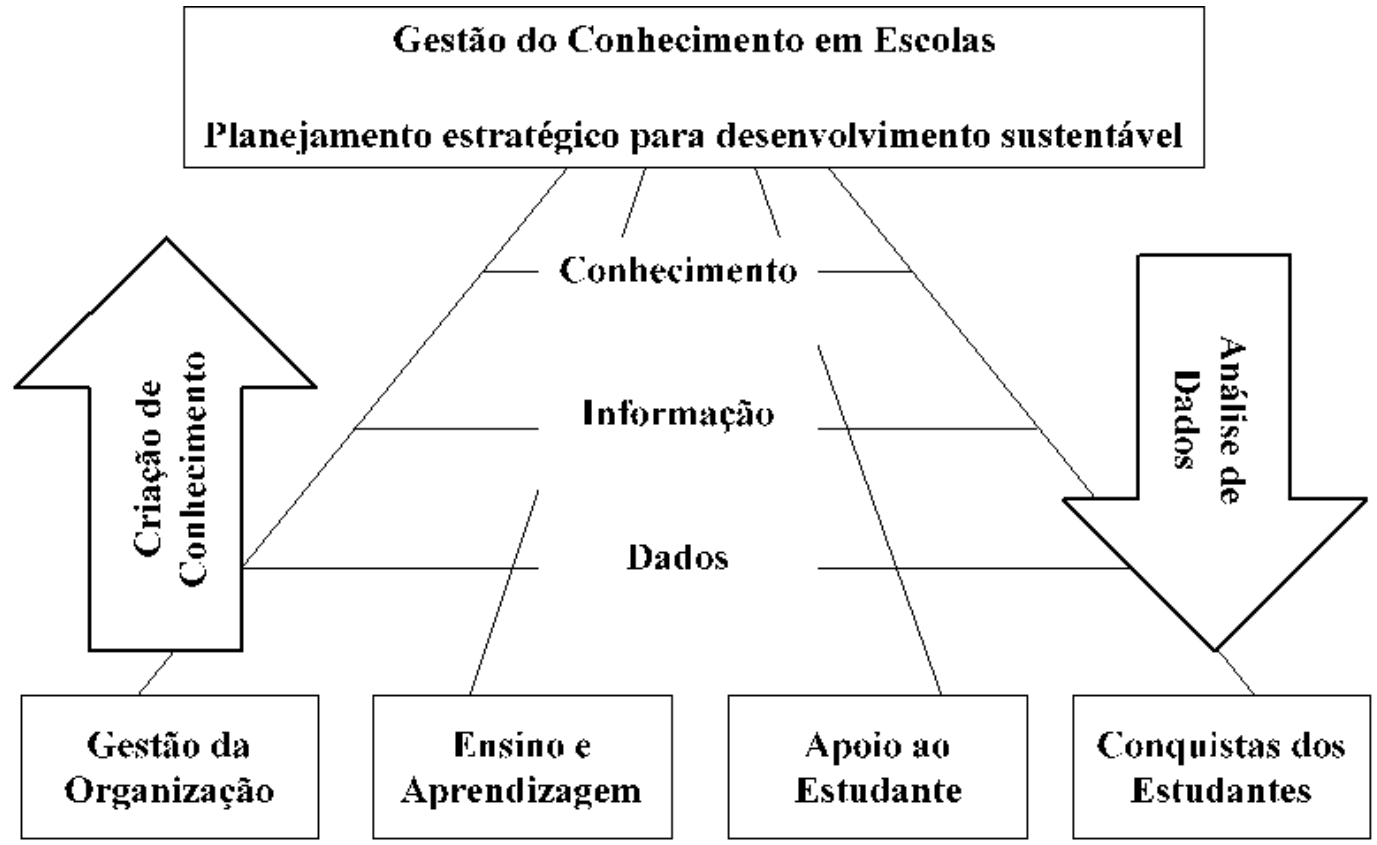

Fonte: Adaptado de Cheng (2013, p.345).

Uma grande quantidade de dados pode ser obtida e registrada pela observação das atividades corriqueiras do ambiente educacional, como mostra a Figura 1. Tais dados, a partir de seleção e análise pelos profissionais envolvidos, podem transformar-se em informações e gerar novos conhecimentos, que podem ser utilizados no processo educacional, de acordo com um planejamento estratégico pré-estabelecido.

A valorização da aprendizagem e da criação de conhecimento na sociedade atual tem obtido suporte do desenvolvimento das tecnologias de informação e comunicação (TIC), com público crescente, tanto em organizações voltadas ao mundo dos negócios quanto as de ensino (COUROS, 2003). Entretanto, no ambiente educacional, ferramentas de gestão do conhecimento ainda são escassas, no qual o método tradicional de ensino está muito presente, sendo um fator limitador da criatividade. 


\section{COMUNIDADE DE PRÁTICA (COP) NO ENSINO SUPERIOR}

A proposta deste trabalho foi realizar um estudo exploratório, por meio de pesquisa bibliográfica, buscando responder a seguinte questão: quais fatores contribuem para a criação, manutenção e fortalecimento de uma CoP no ensino superior? Nesse sentido, procurou-se destacar ideias que contribuam para a aplicação da teoria de comunidade de prática no ensino superior, tanto com a finalidade de preparar estudantes para a prática profissional, quanto para a melhoria do próprio trabalho docente, valorizando as relações entre os pares e a experiência profissional. Dessa forma, é desejável que os grupos formados para apoiar e desenvolver cada atividade da CoP tenham predisposição a um trabalho multidisciplinar, considerando a complexidade da prática.

Na área da engenharia, a atuação profissional no mundo contemporâneo, altamente informatizado, articulado e competitivo, exige competências que vão além dos conteúdos técnicos específicos. A criatividade e o relacionamento interpessoal são aspectos muito importantes e devem ser valorizados. Em busca de inovação tecnológica novas propostas pedagógicas para formação profissional são necessárias, tendo em vista que os métodos tradicionais não atendem as demandas atuais, apesar de ainda muito presentes nas instituições de ensino. Para modificar essa realidade é necessário que professores de cursos de engenharia se aperfeiçoem no domínio das modernas teorias de aprendizagem, além de conhecer a realidade profissional. De acordo com Laudares e Ribeiro (2000, p.497), no contexto da sociedade atual,

A formação acadêmica do engenheiro certamente não mais se faz,
com exclusividade, pelas ciências exatas e sua
qualificação/requalificação em serviço requer novos saberes, com
relações sociais originadas da posição flexível em face das demandas
da abertura dos processos de trabalho.

Newswander e Borrego (2009) afirmam que os métodos pedagógicos tradicionais precisam ser revistos, pois não oferecem os melhores meios de desenvolver nos estudantes habilidades para um trabalho colaborativo, frente o aumento da demanda de profissionais em ambientes pouco estruturados. Para atrair e reter os melhores estudantes, melhores técnicas de aprendizagem precisam ser criadas e adotadas por meio de novos métodos de ensino. Para esses autores, que propuseram uma comunidade 
de prática denominada Journal Club, o modelo de comunidade de prática é um bom caminho para lidar com situações de aprendizagem interdisciplinares, tanto entre estudantes quanto entre docentes, em vários níveis de conhecimento.

A Journal Club é uma comunidade de prática constituída de docentes, estudantes e parceiros da indústria que se reúnem para estudar e discutir, com base na literatura atual, um problema previamente definido. O problema é caracterizado por uma pedagogia que permite desenvolver o pensamento crítico e criativo, de acordo com Newswander e Borrego (2009), características fundamentais à formação profissional, conforme consta as diretrizes curriculares nacionais em diversos cursos, notadamente o de engenharia (CNE, 2002).

Nesse sentido, a criação de uma CoP multidisciplinar, com profissionais da área de formação específica e da área pedagógica pode indicar um bom caminho para a viabilização de mudanças no ensino superior, com o compartilhamento e criação de conhecimento. "Os principais benefícios das CoPs são as aprendizagens entre os pares, a resolução de problemas e pensamento crítico, acúmulo de conhecimento e colaboração.” (NEWSWANDER; BORREGO, 2009, p.563, tradução nossa).

Em tal contexto, duas questões podem ser destacadas: a frequente ausência de formação pedagógica por professores das algumas áreas científicas e tecnológicas e a atuação dos professores novatos. Newswander e Borrego (2009) afirmam que há vários trabalhos sobre CoPs em negócios e indústrias, indicados em publicações de diversos estudos de caso, entretanto, faltam estudos na educação superior, no que este artigo procura contribuir.

Kolikant et al. (2006) desenvolveram uma pesquisa sobre comunidades de prática por meio de cinco equipes transdisciplinares, cada uma composta por dois profissionais, um cientista da área da educação e outro docente da área de engenharia, com o objetivo de elaborar estratégias para a melhoria da aprendizagem em engenharia biomédica. Para isso, propuseram módulos de ensino em conteúdos específicos, tendo em vista o curso como um todo. A Figura 2 representa esquematicamente o processo de desenvolvimento de cada módulo, bem como o papel assumido pelo cientista da área de educação e aquele assumido pelo docente da área de engenharia. $\mathrm{O}$ estudo descreve a dinâmica da relação colaborativa entre os dois profissionais. 
Figura 2 - Processo de desenvolvimento de módulos de aprendizagem em temas de engenharia, o papel de cientistas da educação e o domínio dos especialistas

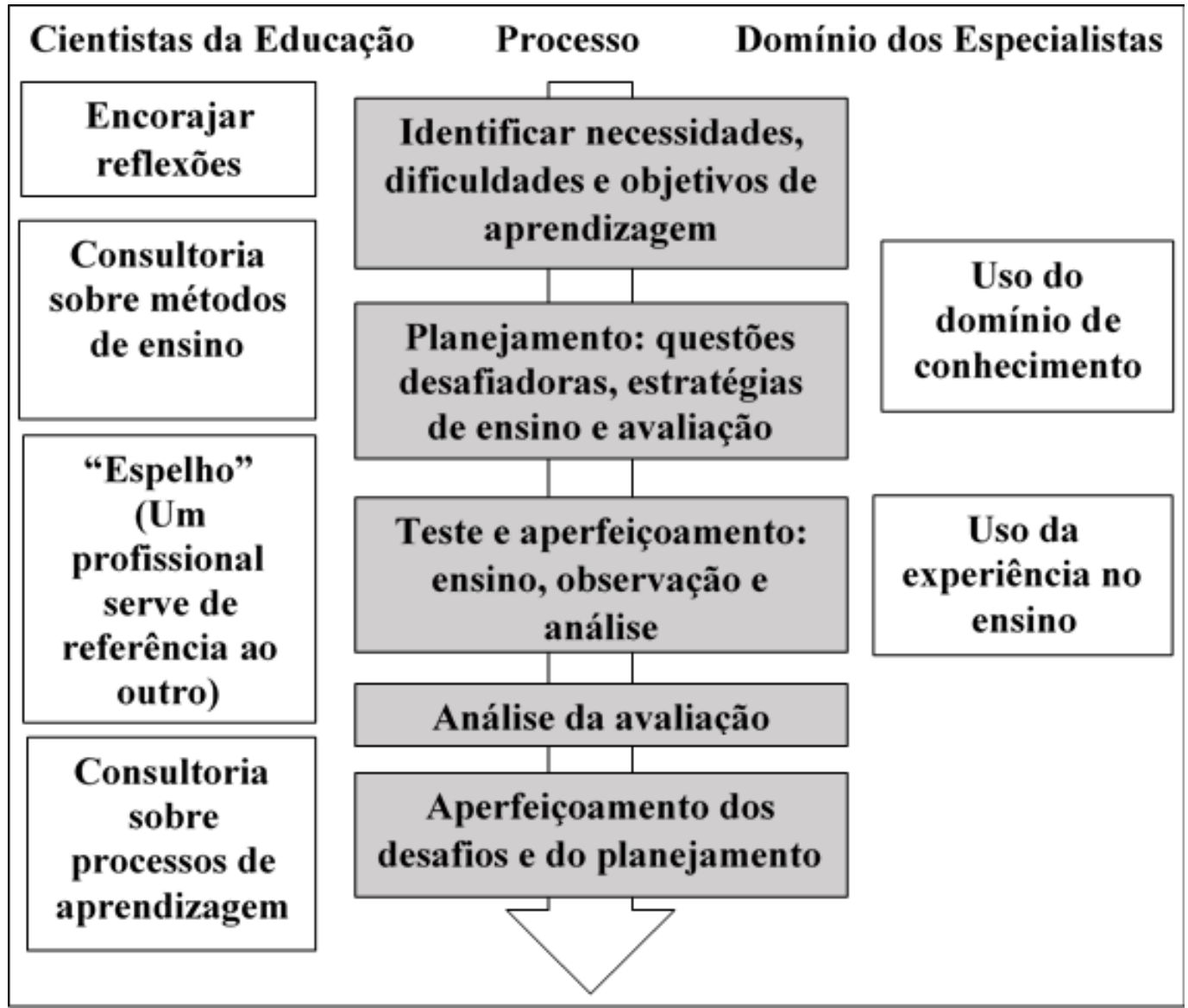

Fonte: Adaptado de Kolikant et al. (2006).

Para Kolikant et al. (2006), a estratégia de começar uma CoP refletindo sobre a realidade que cada profissional enfrenta, sem a cobrança de encontrar soluções imediatas, possibilitou o conhecimento do mundo profissional de cada um pelo outro colega, do vocabulário próprio e das experiências práticas, além dos aspectos teóricos da própria comunidade de prática que foi estabelecida e denominada VaNTH. Tal comunidade de prática envolveu equipes do centro de bioengenharia e educação das universidades de Vanderbilt, Northwestern, Texas e da Divisão de Harvard e do MIT (Massachusetts Institute of Technology) da área de ciências da saúde e tecnologia, buscando desenvolver melhoria na compreensão da bioengenharia entre os estudantes e os melhores métodos para ajudá-los a aprender.

A organização do trabalho em equipe para o desenvolvimento de um projeto, muitas vezes, é realizada de maneira intuitiva, sem incorporar métodos inovadores de gestão. Sendo assim, Zemke e Zemke (2014) afirmam que professores novatos não 
incorporam métodos pedagógicos inovadores oriundos das pesquisas, mas, geralmente, atuam baseados em sua própria experiência como estudante.

As disciplinas associadas às ciências exatas, por exemplo, física, matemática e algumas específicas da área tecnológica, normalmente são desenvolvidas com foco no ensino, por meio de transferência de conhecimento, do professor ao aluno, ou ainda pela resolução de problemas. Uma forma diferente de trabalho ocorre em disciplinas da área das ciências humanas e sociais, com a valorização das concepções dos estudantes, ou seja, com foco na aprendizagem, "uma abordagem muito mais interativa" (ZEMKE; ZEMKE, 2014, p.3, tradução nossa).

Apesar disso, Zemke e Zemke (2014) afirmam que é possível criar uma comunidade de prática entre professores de engenharia, mesmo em um cenário desafiador, com sobrecarga de trabalho, conflitos escolares e divergências em relação à aprendizagem de novas estratégias pedagógicas. Na comunidade de prática por eles criada e investigada, denominada "Clube de Ensino", obtiveram um ambiente seguro para o compartilhamento de conhecimento, com baixos custos sociais e materiais, mas alto benefício potencial.

Cornélio, Abreu e Costa (2010) desenvolveram uma pesquisa com o objetivo de criar um modelo para estreitar o relacionamento entre a universidade e empresas. $\mathrm{O}$ modelo adotou como base teórica a dinâmica de uma comunidade de prática. Para esses autores, a criação do modelo denominado "Espaço Interativo" conseguiu estimular a aproximação do conhecimento entre universitários e as práticas do mundo dos negócios, nos dois sentidos, buscando condições favoráveis à inovação tecnológica. Entretanto, eles afirmam que a falta de recursos financeiros para atividades dessa natureza é a principal dificuldade enfrentada na manutenção da equipe de apoio, sendo necessário firmar parcerias para a viabilização do projeto.

Strazdins (2008) elaborou uma pesquisa para aplicar e avaliar princípios da CoP em projetos de tecnologia da informação a nível de pós-graduação, em uma universidade da Austrália. O principal objetivo foi propiciar um ambiente que forneça experiências favoráveis aos estudantes no desenvolvimento dos seus projetos, melhorando o desempenho e a compreensão dos estudantes sobre questões de pesquisa estudadas na CoP. Alguns indicadores foram utilizados para avaliar a eficiência do projeto: as percepções dos estudantes em relação à melhoria da compreensão e prática sobre métodos de pesquisa, com a participação na $\mathrm{CoP}$; se a participação reflete na 
avaliação formal dos projetos e se há evidências de boas práticas nos grupos participantes da CoP.

O estudo de Strazdins (2008) mostrou que os princípios da CoP podem ser aplicados com sucesso no desenvolvimento de projetos de tecnologia da informação, com o objetivo de ensinar e aprender sobre princípios e habilidades gerais de pesquisa, em nível de pós-graduação. A CoP também se mostrou bastante eficaz quanto ao aproveitamento dos recursos físicos e financeiros devido a aprendizagem coletiva dos estudantes, visando atingir os objetivos de cada projeto. Além disso, os estudantes são mais motivados pelo contexto das discussões sobre os projetos reais, pois relacionam os princípios gerais de pesquisa com sua experiência atual. O processo de revisão pelos pares também contribui para a motivação e percepção da melhoria quanto a compreensão e habilidades de estratégias de pesquisa, por meio das sessões da CoP.

Blanton e Stylianou (2009) analisaram o engajamento de professores novatos ao corpo docente veterano no ensino superior, em universidades norte americanas, à luz da teoria sobre comunidade de prática. Os resultados indicaram que tal engajamento traz diversos desafios, como a necessidade do desenvolvimento de uma cultura profissional e de engajamento entre o corpo docente veterano e os professores novatos, em determinada disciplina; a necessidade de coordenar esse desenvolvimento profissional, refletindo sobre a cultura profissional e a necessidade de uma linguagem comum para mediar essa reflexão.

Zemke e Zemke (2014, p.13, tradução nossa), baseados em resultados de pesquisa, estabeleceram algumas recomendações, apontadas a seguir, para a criação de uma CoP, principalmente em pequenas escolas de engenharia.

1. Estruturar discussões acerca das necessidades imediatas dos docentes em suas aulas gera maior engajamento do que discussões acerca de uma referência. Apesar da perda de profundidade teórica, há um aumento na aplicação de ideias.

2. Um facilitador com habilidade em moderar a interação em pequenos grupos é importante. Analogamente, designar alguém para gerenciar a logística das reuniões é vital para a manutenção da CoP. As reuniões e discussões não ocorrem sem apoio intencional.

3. Proteger a confidencialidade das discussões dos processos de promoção e mandato em determinado cargo, também é vital. Os membros não são transparentes se eles percebem que compartilhar conhecimento pode afetar suas avaliações institucionais. 
O Quadro 1 apresenta uma síntese das principais ideias, aqui denominadas de boas práticas, que auxiliam na criação, manutenção e fortalecimento de uma comunidade de prática.

Quadro 1 - Síntese das ideias centrais (boas práticas) para a criação, manutenção e fortalecimento de uma Comunidade de Prática (CoP).

- Os membros são agrupados de forma voluntária e informal pela emoção vinculada ao tema e pela identificação com a especialidade do grupo. Os membros não devem ser designados e a liderança deve ser estabelecida informalmente.

- Por meio das atividades da CoP, constrói-se um sentido de pertencimento entre seus membros, caracterizado pelo compartilhamento de interesses.

- A estreita ligação entre as discussões e o trabalho em sala de aula aumenta o 2008. engajamento, sua relevância e o investimento pessoal.

- Não deve haver conflitos de interesses entre os membros da CoP, embora possam envolver conflitos de interesses dentro da organização ou nas relações sociais na rede dos membros.

- As atividades de produzir, armazenar, compartilhar e utilizar conhecimento por meio das interações entre seus membros dependem das características culturais e do ambiente (contexto).

- A motivação interna (emoção positiva) não mostrou influência significativa sobre as atividades da CoP on-line, como ocorre com a CoP off-line.

- Há preferência pela resolução de problemas organizacionais por meio de interações off-line, em vez de on-line. A consciência de grupo é um fator de forte influência nas atividades off-line, superando a influência do compartilhamento, criação e uso do

JANG; KO, conhecimento por meio de atividades on-line.

- O sucesso das CoP on-line depende muito mais do apoio organizacional do que das off-line, devido a maior dificuldade de formação de uma consciência de grupo.

- Atividades CoP on-line podem dar suporte às atividades off-line, facilitando o registro do conhecimento no sistema de informação, principalmente dos problemas resolvidos. Isto pode contribuir para as atividades em outros setores da organização, podendo gerar resultados inovadores.

- Há menos engajamento com tópicos de livro texto do que com conversas desestruturadas.

ZEMKE;

ZEMKE,

2014; JANG;

KO, 2010; STRAZDINS,
ZEMKE;

ZEMKE,

2014.

- A melhoria na comunicação entre os membros e habilidades de gerenciamento de projetos são aspectos suficientes para estabelecer uma CoP eficiente e coesa.

STRAZDINS,

- A CoP pode proporcionar a maximização de recursos de aprendizagem por meio de 2008. trocas de experiências.

Fonte: Elaboração própria.

Para Crede, Borrego e McNair (2010), preparar estudantes para a carreira docente não é uma novidade, mas explicitar a aplicação de comunidades de prática e a literatura relacionada é algo novo. Eles apresentam os resultados de uma pesquisa destinada a analisar a aplicação da teoria sobre CoP em um programa de preparação de docentes na área de engenharia. Os resultados indicam que essa teoria pode contribuir 
significativamente para trazer um aprofundamento adicional aos programas de formação docente. Eles também afirmam que as relações entre os estudantes, que atuaram como assistentes e os docentes experientes contribuíram para equilibrar ensino e pesquisa, ajudando a preparar os novatos para os múltiplos aspectos da carreira docente.

A teoria da $\mathrm{CoP}$ pode contribuir para a educação superior, como destaca Newswander e Borrego (2009, p.569, tradução nossa),

Preparando os empresários, acadêmicos e líderes políticos do futuro devendo incluir um plano bem-pensado para a socialização dentro do campo de estudos. O formato Journal Club, como um exemplo da teoria $\mathrm{CoP}$ em ação, fornece um ambiente acadêmico e social que ajuda os docentes a promover um nível mais profundo de compreensão entre estudantes e os prepara melhor para a vida depois da graduação.

O Quadro 2 apresenta uma síntese dos desafios e algumas sugestões de superação. Pode-se observar que há certa aproximação entre os resultados encontrados na pesquisa bibliográfica e o trabalho de Davenport e Prusak (1999), citado na introdução deste artigo. Nota-se a necessidade de mais pesquisas sobre o desenvolvimento de comunidades de prática em escolas do país, para a adequação e atualização desses quadros no contexto nacional.

Algumas comunidades de prática foram criadas no Brasil, em cursos de licenciatura, principalmente na área da formação de professores de matemática, visando a inserção de professores novatos na realidade da sala de aula, identificação e reflexão sobre os problemas enfrentados, bem como para a atualização dos profissionais em exercício, conforme analisado nos trabalhos de Carvalho (2007), Pamplona e Carvalho (2009), Calceira (2010) e Silva (2010).

Além disso, é possível reconhecer no programa PIBID (Programa Institucional de Bolsas de Iniciação à Docência) (BRASIL, 2008), diversas atividades que podem ser estruturadas e avaliadas segundo os fundamentos teóricos das comunidades de prática. No programa, equipes são formadas congregando estudantes de licenciatura, professores universitários e professores de nível básico, com o objetivo de proporem soluções aos problemas identificados nas escolas de nível básico envolvidas.

Pesquisas desenvolvidas por Benites (2013) e Santos (2015) analisam grupos de trabalho por meio de indicadores que caracterizam as comunidades de prática, na área de formação de professores de matemática e química, respectivamente. Em ambas as pesquisas, os licenciandos também estavam comprometidos com o programa PIBID. Os 
resultados apontam para a importância da formação de comunidades de prática no processo de formação de professores, pois contribuem para a formação de uma identidade entre seus membros, propiciando a elaboração de projetos conjuntos e o compartilhamento de repertórios e materiais didático-pedagógicos. Além disso, estimulam a aproximação do mundo acadêmico com a realidade escolar, criando um ambiente de reflexão sobre as práticas educacionais. 
Quadro 2 - Síntese dos desafios e sugestões de superação para a criação, manutenção e fortalecimento de uma Comunidade de Prática $(\mathrm{CoP})$.

\begin{tabular}{|lll|}
\hline \multicolumn{1}{|c}{ Desafios e sugestães } & \multicolumn{1}{c}{ Fonte } \\
\hline - As CoPs não se ajustam bem às estruturas organizacionais tradicionais. & \\
- São necessários disponibilidade de tempo, energia e apoio para o & & \\
desenvolvimento de atividades CoP. & ZEMKE; ZEMKE, \\
- É necessário um facilitador (remunerado ou recompensado) responsável & 2014; JANG; KO, \\
pela viabilidade dos encontros, moderação das discussões e organização de & $2010 . \quad$ STRAZDINS, \\
materiais necessários às reuniões. Com ele, os demais membros ficam & 2008. & \\
concentrados em suas especialidades. Ele também pode ajudar na transposição & & \\
de ideias dos referenciais teóricos para situações práticas. & \\
\hline
\end{tabular}

- $\quad$ Apoio organizacional para minimizar ou neutralizar os efeitos da perda da exclusividade de um conhecimento como fator de risco na manutenção do emprego, como consequência das atividades da CoP. Necessidade de um ambiente que incentive o compartilhamento de conhecimento.

- $\quad$ Todo conhecimento é considerado formal quando apresentado on-line. Isto faz com que a percepção do risco da perda da exclusividade do conhecimento aumente, inibindo a atividade da CoP. Nas atividades off-line tal percepção é menor, pois a disseminação e o registro do conhecimento são limitados, devido à interação "face a face". Provavelmente a consciência de grupo seja maior nas atividades off-line.

- Quanto maior a lacuna existente entre o conhecimento formal da organização e o conhecimento tácito de seus membros, maior os conflitos devido às assimetrias de poder entre eles e a organização.

- $\quad$ Perda de oportunidades de discussões em temas teóricos relevantes, do ponto de vista do pesquisador da $\mathrm{CoP}$, em detrimento da valorização das reais necessidades dos Professores.

- Restrição de tempo das reuniões.

JANG; KO, 2010.

- $\quad$ Não está claro se reuniões estruturadas tendo como base tópicos de um livro texto desestimula o engajamento ou são outros os fatores desestimulantes, como doença, falta de continuidade, sobrecarga de trabalho, cansaço etc.

- Não foram observadas propostas de práticas inovadoras de sala de aula a partir de estudos teóricos nesse sentido. Este aspecto foi considerado menos

ZEMKE; ZEMKE, relevante que as próprias propostas dos membros.

- A interdisciplinaridade é um obstáculo na discussão de questões de conteúdo específico.

- Condições para a sustentabilidade de uma CoP: uniformidade de propostas visando benefício mútuo e rápido feedback (avaliação formativa) sobre os projetos propostos.

- $\quad$ Em comunidades multidisciplinares é necessário o compartilhamento do vocabulário para uma efetiva comunicação.

2014.

ZEMKE; ZEMKE, 2014. STRAZDINS 2008.

STRAZDINS, 2008.

- Ambiente que propicie a negociação quanto aos métodos de trabalho, com foco na aprendizagem.

- $\quad$ A implementação de CoPs no ensino superior pode começar com os professores concentrando esforços por meio de seminários sobre assessoria em relacionamentos, interações e engajamento.

KOLIKANT, Y. B. D. et al., 2006.

NEWSWANDER;

BORREGO， 2009; BLANTON; STYLIANOU, 2009.

Fonte: Elaboração própria. 


\section{CONSIDERAÇÕES FINAIS}

Muitas informações são geradas no ambiente educacional, entretanto, não são organizadas, registradas, disseminadas ou recuperadas, considerando sua possível utilização na melhoria das condições de aprendizagem e do desempenho dos estudantes e demais profissionais envolvidos no processo.

O conhecimento da estrutura, formação e dinâmica das comunidades de prática pode auxiliar no processo de aprendizagem de estudantes e professores em nível superior, considerando a instituição de ensino como um local de criação de conhecimento e de intensa atividade social. Assim, devem-se estimular as interações entre os membros da comunidade, entre organizações e também com pessoas externas a elas. Além disso, é possível considerar que as habilidades desenvolvidas no ambiente acadêmico podem contribuir para a formação profissional do estudante, pensando em sua atuação futura em determinadas instituições de ensino, pesquisa ou do mundo dos negócios, públicas ou privadas, com ênfase na criação de conhecimento, produtos e processos inovadores.

A CoP também pode contribuir para a melhoria da prática docente, pois inspira sentimentos de confiança, receptividade, pertencimento e responsabilidade, facilitando o compartilhamento de técnicas e métodos que estão sendo aprendidos e/ou melhorados. "Uma CoP propicia oportunidades para formar redes, dar apoio e sustentar relações" (ZEMKE; ZEMKE, 2014, p.4, tradução nossa).

Dois fatores influenciam significativamente o desempenho das comunidades de prática, o compromisso assumido na relação entre seus membros e a eficiência, desenvolvida pela CoP, na resolução de problemas (JANG; KO, 2010).

Uma questão que pode ser levantada para pesquisas futuras é se o conhecimento da teoria sobre comunidade de prática, de fato, contribui efetivamente para sua criação, manutenção e fortalecimento em cursos no ensino superior, principalmente nas áreas de ciências exatas e tecnologia. As pesquisas apontam no sentido positivo em relação a melhora nas atividades de ensino e aprendizagem, bem como nos ambientes empresariais. Cabe investigar mais sobre os aspectos da prática profissional no ambiente acadêmico, superando os desafios encontrados.

A literatura internacional apresenta diversos trabalhos bem-sucedidos desenvolvidos por meio de comunidades de prática $(\mathrm{CoP})$. Entretanto, nota-se que esse 
recurso da gestão do conhecimento ainda é pouco explorado nas instituições de ensino no Brasil. Talvez existam em atividade diversas comunidades que lidam com temas pertinentes ao ensino superior, porém poucas pesquisas são encontradas. Assim, este artigo procura apontar alguns delineamentos e desafios para sua utilização, visando à superação das dificuldades encontradas no ensino superior para sua implantação, envolvendo tanto os professores quanto estudantes, em relação à aprendizagem de conteúdo, desenvolvimento de projetos ou habilidades práticas inerentes aos cursos, principalmente aqueles relacionados às ciências exatas e tecnologia.

\section{COMMUNITY OF PRACTICE IN HIGHER EDUCATION}

$\overline{\text { ABSTRACT: The goal of this study is to analyze the insertion in higher education a }}$ knowledge management framework called Community of Practice (CoP) or Professional Learning Community. For this, a bibliographic research was made which sought to answer the following question: what factors contribute to the creation, maintenance and strengthening of a CoP in higher education? The results indicate that the inclusion of a CoP in higher education depends on several factors, including the enhancement of social relations among its members, institutional support as a way to overcome the loss of exclusivity of knowledge through the recording of information and the need a facilitator who enables and organize the meetings and encourages attendance at meetings. The community of practice in higher education can take place between professors of specific areas and professors of pedagogical area, and among students, or as a combination of these actors. Moreover, it is possible to involve external parties, such as professionals linked to public or private companies. Several international studies have shown successful results, however, few studies can be found in Brazil.

KEYWORDS: Community of Practice (CoP). Knowledge management. Higher education.

\section{REFERÊNCI AS}

BENITES, V. C. Formação de professores de matemática: dimensões presentes na relação PIBID e Comunidade de Prática. 2013. 247f. Dissertação (Mestrado) - Instituto de Geociências e Ciências Exatas, Universidade Estadual Paulista, Rio Claro, 2013.

BLANTON, M. L.; STYLIANOU, D. A. Interpreting a Community of Practice perspective in discipline-specific professional development in Higher Education. Innovative Higher Education, New York, v.34, n.2, p.79-92, jun. 2009.

BRASIL. CAPES. PIBID - Programa Institucional de Bolsas de Iniciação à Docência. Brasília: Ministério da Educação, 2008. Disponível em: 
<http://www.capes.gov.br/educacao-basica/capespibid/pibid>. Acesso em: 30 nov. 2015.

CALDEIRA, J. S. Um estudo sobre o pensamento algébrico em uma comunidade de prática de formação de professores de matemática. 2010. 127f. (Mestrado em Ensino de Ciências e Educação Matemática) - Centro de Ciências Exatas, Universidade Estadual de Londrina, Londrina, 2010.

CARVALHO, V. Mathematics Education and Society (MES): a constituição de uma comunidade de prática científica internacional. 2007. 409p. (Doutorado em Educação) Faculdade de Educação, Universidade Estadual de Campinas, Campinas, 2007.

CHENG, E. C. K. Applying knowledge management for school strategic planning. KEDI Journal of Educational Policy, Seoul, v.10, n.2, p.339-356, 2013. Disponível em: <http://eng.kedi.re.kr/khome/eng/kjep/pubList.do\#>. Acesso em: 10 out. 2015.

CONSELHO NACIONAL DE EDUCAÇÃO [CNE]. Institui Diretrizes Curriculares Nacionais do Curso de Graduação em Engenharia. Resolução CNE/CES 11, de 11 de março de 2002. Diário Oficial da União: República Federativa do Brasil, DF, 11 mar. 2002. Disponível em: <http://portal.mec.gov.br/cne/arquivos/pdf/CES112002.pdf>. Acesso em: 01 nov. 2015.

CORNÉliO, N. A. G.; ABREU, A. F. de; COSTA, E. O. Espaço interativo: modelo de relação universidade-empresa baseada em comunidades de prática. Ciência da Informação, Brasília, v.39, n.1, p.9-20, 2010. Disponível em: <http://www.scielo.br/pdf/ci/v39n1/v39n1a01.pdf>. Acesso em: 03 nov. 2015.

COUROS, A. Communities of practice: a literature review. 2003. Disponível em: <http://www.tcd.ie/CAPSL/_academic_practice/pdfdocs/Couros_2003.pdf>. Acesso em: 04 out. 2015.

CREDE, E.; BORREGO, M.; MCNAIR, L. D. Application of community of practice theory to the preparation of engineering graduate students for faculty careers. Advances in Engineering Education, Washington, v.2, n.2, p.1-22, 2010. Disponível em: <http://advances.asee.org/wp-content/uploads/vol02/issue02/papers/aee-vol02-issue02p04.pdf>. Acesso em: 10 out. 2015.

DAVENPORT, T. H.; PRUSAK, L. Working knowledge: how organizations manage what they know. 2000. Disponível em: <http://www.kushima.org/is/wpcontent/uploads/2013/09/Davenport_know.pdf >. Acesso em: 12 out. 2015.

Transferência do conhecimento. In: Conhecimento empresarial. Rio de Janeiro: Campus, 1999. p.107-128.

FENWICK, T.; EDWARDS, R. Networks of knowledge, matters of learning, and criticality in higher education. Higher Education, [S.1], v.67, n.1, p.35-50, 2014.

FERREIRA, A. A.; SILVA, B. D. Comunidade de prática on-line: uma estratégia para o desenvolvimento profissional dos professores de história. Educação em Revista, Belo 
Horizonte, v.30, n.1, p.37-64, 2014. Disponível em:

<http://www.scielo.br/pdf/edur/v30n1/a03v30n1.pdf>. Acesso em: 03 nov. 2015.

JANG, H.; KO, I. The factors influencing the online/off-line activities of the CoP (Community of Practice) and the results: focusing on perceived risk of losing uniqueness of one's own knowledge through CoP activity. In: INTERNATIONAL CONFERENCE ON SYSTEM SCIENCES, 43., 2010, Hawaii. Proceedings... Hawaii: IEEE CONFERENCE PUBLICATIONS, 2010. p.1-12.

KOLIKANT, Y. B. D. et al. The emergence of a community of practice in engineering education. New Directions for Teaching and Learning, San Francisco, n.108, p.7-16, 2006. Disponível em: <http://doi.wiley.com/10.1002/tl.252>. Acesso em: 21 out. 2014.

LAUDARES, J. B.; RIBEIRO, S. Trabalho e formação do engenheiro. Revista Brasileira de Estudos Pedagógicos, Brasília, v.81, n.199, p.491-500, 2000. Disponível em: <http://rbep.inep.gov.br/index.php/RBEP/article/viewFile/135/135>. Acesso em: 16 out. 2014.

NEWSWANDER, L. K.; BORREGO, M. Using journal clubs to cultivate a Community of Practice at the Graduate Level. European Journal of Engineering Education, Oxfordshire, v.34, n.6, p.561-71. 2009. Disponível em: <http://www.tandfonline.com/doi/abs/10.1080/ 03043790903202959>. Acesso em: 01 nov. 2015.

NONAKA, I.; TOYAMA, R. The knowledge-creating theory revisited knowledge creation as a synthesizing process. Knowledge Management Research \& Practice, Basingstoke, n.1, p.2-10, 2003. Disponível em: <http://www.palgravejournals.com/kmrp/journal/v1/n1/pdf/8500001a.pdf>. Acesso em: 18 out. 2015.

PAMPLONA, A. S.; CARVALHO, D. L. O ensino de estatística na licenciatura em matemática: a inserção do licenciando na comunidade de prática dos professores de matemática. Bolema, Rio Claro, v.22, n.32, p.47-60, 2009.

SANTOS, V. C. A formação de professores em comunidades de prática: o caso de um grupo de professores de química em formação inicial. 2015. 451f. Tese (Doutorado em Ciências) - Universidade de São Paulo, São Paulo, 2015.

SILVA, H. Uma caracterização do Centro de Educação Matemática - CEM (19841997) como uma comunidade de prática de formação continuada de professores de matemática. Bolema, Rio Claro, v.23, n.35, p.185-218, 2010.

STRAZDINS, P. Applying the community of practice approach to individual IT projects. In: CONFERENCE ON AUSTRALASIAN COMPUTING EDUCATION, 10., 2008, Wollongong. Proceedings... Wollongong: Australian Computer Society, 2008. p.137-146. Disponível em: <http://dl.acm.org/citation.cfm?id=1379263>. Acesso em: 10 out. 2015.

UNITED NATIONS OF DEVELOPMENT PROGRAMME [UNDP]. Knowledge management strategy framework: 2014-2017. 2014. Disponível em:

<http://www.undp.org/content/dam/undp/library/capacity- 
development/English/UNDP\%20Knowledge\%20Strategy\%20Report\%2025022\%20LR\%202,7MB.pdf >. Acesso em: 24 out. 2015.

WENGER, E. Communities of practice: a brief introduction. 2015. Disponível em: <http://wenger-trayner.com/wp-content/uploads/2015/04/07-Brief-introduction-tocommunities-of-practice.pdf >. Acesso em: 14 set. 2015.

ZEMKE, D. L.; ZEMKE, S. C. Using a Community of Practice to diffuse instructional improvements into the classroom. In: AMERICAN SOCIETY FOR ENGINEERING EDUCATION ANNUAL CONFERENCE \& EXPOSITION, 121., 2014, Indianapolis. Proceedings... Indianapolis: American Society for Engineering Education, 2014. Disponível em: <http://www.asee.org/public/conferences/32/papers/8443/view>. Acesso em: 11 out. 2015. 